\title{
In-vitro culture protocol development for early stage zebrafish ovarian follicles
}

\author{
Siji Anil ${ }^{1}$, David Rawson ${ }^{2}$ and Tiantian Zhang ${ }^{3}$ \\ ${ }^{1}$ NCBS National Centre for Biological Sciences, Tata Institute of Fundamental Research, \\ Bangalore, India \\ ${ }^{2}$ iBEST Institute of Biomedical and Environmental Science and Technology, University of \\ Bedfordshire, Luton, UK \\ ${ }^{3}$ Faculty of Science and Technology, Bournemouth University, Poole, UK
}

Studies on in vitro culture of early stage zebrafish ovarian follicles in ovarian tissue fragments is reported here for the first time. Although several in vitro maturation procedures developed for late stage ovarian follicles have been reported, information on in vitro maturation of early stage ovarian follicles has been very limited. The main aim of the present study was to develop an in vitro culture protocol for stage I and stage II zebrafish ovarian follicles embedded in ovarian tissue fragments. The effect of concentration of growth hormones such as human chorionic gonadotropin and follicle stimulating hormones, and growth supplements such as Foetal Bovine Serum and Bovine Serum Albumin were studied. The results showed that early stage zebrafish ovarian fragments containing stage I and stage II follicles can be cultured in vitro for $24 \mathrm{~h}$. Stage I and II ovarian follicles can grow to the size of stage II and stage III ovarian follicles respectively following incubation in $20 \%$ FBS and $100 \mathrm{mIU} / \mathrm{ml} \mathrm{FSH}$ in $90 \% \mathrm{~L}-15$ medium at $28^{\circ} \mathrm{C}$. The follicle growth from stage I to stage II and from stage II to stage III were confirmed using the molecular markers such as P450aromA and vtg1 genes respectively. The results from this study demonstrate that early stage ovarian follicles can be matured in vitro from stage I to stage II and from stage II to early stage III, the study also provides a better understanding of the mechanisms of zebrafish ovarian follicles maturation in vitro. 